\title{
The management of somatisation
}

\author{
Graeme C. Smith
}

Somatisation remains a matter of bewilderment in medicine. Medical students have trouble defining it, psychiatry trainees wilt in its presence, and all doctors are prone to become entangled in it. The current spate of reviews on the topic is appropriate (Mayou et al, 1995a; Barsky, 1996; Gill \& Bass, 1996). There is a great need for those required to manage patients with 'medically unexplained symptoms' to be informed sufficiently to take a stance on the theoretical issues involved and develop appropriate management plans.

Faced with a display of physical symptoms for which neither lesion nor pathophysiological cause can be substantiated, the practitioner has the choice of admitting ignorance, suspecting fraud, or evoking the concept of psychogenesis, mind-made disorder, as an explanatory model. How well is the modern doctor, let alone the patient, prepared to deal with models of uncertainty, fraudulence and mind? Feelings of impotence are invoked on the part of the doctor, and feelings of responsibility, even blame, on the part of the patient. The doctor-patient relationship, on which resolution will depend, is under grave threat in the presence of medically unexplained physical symptoms, of which somatisation is an example.

Just as Freud, in offering a model for the understanding of symptom formation and a psychological mode of treatment, liberated both patient and doctor from the tyranny of unexplained symptoms in this century, so too a new generation of empirical studies are providing rational treatment models that are reempowering practitioners to deal with such problems as they now present (Smith et al, 1995). This paper aims to trace this evolution and identify guidelines that will facilitate practice.

\section{Definition}

A widely quoted definition of somatisation is that of Lipowski (1988):
"The tendency to experience and communicate somatic distress and somatic symptoms unaccounted for by relevant pathological findings, to attribute them to physical illness, and to seek medical help for them."

An extreme example illustrates the three elements of this constricted and highly digested definition; the experiential, the cognitive and the behavioural.

Ms G is a 45-year-old patient of virtually every unit in the hospital except Obstetrics, and all of her complaints use the language of the body. She has had a spinal fusion, a colostomy, an ileal conduit for her ureters, and removal or restoration of a number of abdominal organs. She is on large doses of many drugs, and claims allergy to many others, as well as to the environment. When admitted under one unit, she telephones the secretaries of other units to coordinate their consultations. She keeps a voluminous diary of what consultants say, and of her test results, and the consultants use this as a means of communication with each other. She brings her own medication, and directs the writing of her drug chart, and monitors its execution. Despite this, she is the apparent victim of much miscommunication and adverse drug reactions, and the cause of much anger and guilt among staff.

\section{The wider perspective}

Lipowski's definition, and the case illustration, are extremes. A broader perspective is required to avoid marginalising somatisation as something bizarre and unusual. It is essential to grasp the point that somatisation as a phenomenon is not equivalent to, nor confined to, the more severe cases defined by the ICD-10 category 'somatisation disorder' (World Health Organization, 1992) or its DSM-IV equivalent (American Psychiatric Association, 1994). Rather, somatisation is an ubiquitous tendency present in many cultures (Kirmayer et al, 1994), and more likely than not to be a comorbid

Graeme Smith, FRANZCP, is Professor of Psychological Medicine in the Monash University Department of Psychological Medicine and the Consultation-Liaison Psychiatry Research Unit at Monash Medical Centre, 246 Clayton Rd, Clayton VIC 3168, Melbourne, Australia. This Unit is developing diagnostic and screening instruments for cases of physical-psychiatric comorbidity and somatisation. Professor Smith is Chairperson of the Section of Consultation-Liaison Psychiatry of the Royal Australian and New Zealand College of Psychiatrists. 
feature of other psychiatric disorders and physical disorders (Bass, 1994).

Somatisation is not confined to communication with health care professionals. It forms part of everyday communication. It is how humans think about themselves, and is reflected in their cultural productions:

" He first deceased; she for a little tried

To live without him, liked it not and died."

Sir Henry Wotton (1642)

Is somatisation a medical matter at all? Or is it a sociological phenomenon best understood as playing out the sick role, which draws on somatic and mental models but which does not necessarily imply disorder of either? This post-modern reading commands attention. Nevertheless, in Western culture somatising people often present to doctors, and some are referred to psychiatrists, who must deal with them rationally.

When patients come to psychiatrists we expect to hear mental symptoms; what we get with somatisers are symptoms of the body. Value judgements appear; we regard the somatiser as having opted for a lower order metaphor. The derogatory use of the term hysteric epitomises this. Why is the body so important to patients in general and somatisers in particular? Why are bodily symptoms chosen as the vehicle for expression rather than externally directed behaviour or mental anguish?

History gives some clues. The new wave of scholarly accounts of the history of psychiatry remind us that Western psychiatry embraced psychological thinking only a century ago (Berrios \& Porter, 1995). Psychiatry invented its psychological language at that time. For instance, it borrowed the physical term 'depression' to name the affective component of its newly conceived mood disorder, and it purloined the terms and concepts of 'neurosis' and 'anxiety' from physicians, transforming their clinical meaning from a physical to a psychological one. While lay people may long have had an inherent sense of how intimately social and physical events are related, they have not necessarily adopted the psychogenic model; that is, they find it hard to share the view that mind processes can cause physical symptoms in a way that does not have disease process as an intermediary. Those from other cultures may never have had the chance to be exposed to such notions.

Somatisation is therefore likely to be part of the phenomenology of all illness. It occasionally (and dramatically) presents as a pure and acute psychosocial metaphor, as in hysterical conversion, but most often it is part of a complex biopsychosocial web which health professionals must unravel
(Hiller et al, 1995; Mayou, 1996; Robbins \& Kirmayer, 1996; Rogers et al, 1996). This is captured in the concept of Bass \& Murphy (1995) that chronic somatisation is like a personality disorder. This has important implications for management.

\section{Origins of the term}

Changes in the way that somatisation has been conceptualised and defined add to the bewilderment. The word somatisation dates from the height of the psychosomatic movement in North America in the 1940s, when Stekel and Menninger used it as a name for an unconscious defence (Menninger, 1947). But it was inherent in Freud's revolutionary concept of the psychogenesis of neurotic symptoms through a direct physiological reaction or a symbolic conversion. In this vein, Lipowski (1968) originally postulated a continuum of somatisation reactions from the "conversion and hypochondriacal disorders" to the "psychophysiological disorders." The somatic components of anxiety disorders and depression, and disorders of sleep, eating and sexual function all came within his concept of somatisation, as did factitious disorders. While all of these latter examples of expression of psychogenic symptoms in physical terms are excluded from Lipowski's later definition of somatisation, their mention serves to remind us how ubiquitous such expression is, as illustrated by the following case:

A 65-year-old Greek widow presented complaining of chest pain. She had just had a call from her only daughter saying that she was returning to Greece to live. The patient's husband had died of a myocardial infarction two years previously, and she then moved to a retirement home where she had been unhappy. Her family practitioner had been treating her for mixed anxiety and depression with a low dose of a tricyclic antidepressant.

Are we dealing with myocardial infarction, angina, a psychophysiological reaction, an anxiety attack, worsening depression, hysterical conversion, hypochondriasis or factitious disorder? Whichever one or combination of these it is, the setting is that of abnormal bereavement which has transcultural undertones. In assessing the case, the clinician would also have in mind the concepts of abnormal illness behaviour and alexithymia. Another way of putting it is to say that the clinician will have to decide to what extent physical disease is present, how ill the patient feels, and how all this is being played out as sickness. These concepts represent recent attempts to deconstruct the 
phenomenon of somatisation and reframe it in cultural terms.

Thus the current trend in thinking about somatisation, which focuses only on the circumscribed issue of patients who frequently complain of physical symptoms that either lack demonstrable organic bases, or are judged to be grossly in excess of what one would expect to find on the grounds of objective medical findings, promotes an artificial demarcation. It promotes the mind/body split in situations where an integrated biopsychosocial approach is required.

\section{The ICD and DSM classifications}

Changing terminology in the official classifications adds to the difficulty in comprehending somatisation. The term 'somatoform disorder' was coined by the authors of DSM-III as part of their strategy to remove the term 'neurosis'. In DSM-IV it refers to "the presence of physical symptoms which suggest a general medical condition," and covers acute and chronic conversion conditions. ICD-10 has adopted the term, but limits it to the chronic forms of conversion, using the term 'dissociative' (conversion) to cover the acute forms. It has reintroduced the term 'neurasthenia' to describe chronic fatigue phenomena. This division into acute and chronic presentations is widely supported (Mayou et al, 1995b). Thus those with somatoform disorders somatise in a particular way, but not all somatisers have somatoform disorder.

\section{Alternative terminology}

Other doctors use a different language. The term 'medically unexplained symptoms' is in common use. It seems less pejorative; it does not imply cause, and it acknowledges that a problem exists for which further assessment is appropriate. The term 'functional' has been in use for two centuries. It seems to have arisen as a synonym for physiological in the first half of the 19th century. It was used by Charcot in this way at the end of the 19th century, but as ideogenic notions took hold in the 20th century it embraced these as well. It is thus broader than the term somatoform, and covers both dissociative (conversion) and psychophysiological phenomena, for which ICD-10 uses the awkward term "psychological and behavioural factors associated with disorders or diseases classified elsewhere."

A term used by doctors for much of this century and now part of common language is 'psychosomatic'. Its modern use became consolidated in the 1930s, when Alexander developed Freud's concept of organ neuroses into that of psychosomatic disorders, later to become psychophysiological disorders. The term came to be used to describe the field of medicine concerned with the relationship of biological and psychological factors in general. It entered popular language, and remains in use in a pejorative way. For that reason, psychiatrists specialising in this field now call themselves consultation-liaison psychiatrists rather than psychosomaticists.

\section{Concepts related to somatisation}

Persisting disagreement between patient and doctor about diagnosis or treatment, such as tends to occur in cases of somatisation, has been conceptualised by Pilowsky as abnormal illness behaviour (Pilowsky, 1978). This has proved to be an extremely useful concept in helping us to come to grips with the systems issues involved, and the multifactorial determinants. Its pejorative connotation with respect to the patient has been leavened by Singh's concept of abnormal treatment behaviour (Singh et al, 1981).

The term 'alexithymia' is a neologism which refers to difficulty in feeling or expressing emotions, and in experiencing or expressing fantasies (Taylor, 1987). Like somatisation, it is a popular and seemingly well understood descriptive term and explanatory concept in the field, but it has proved difficult to operationalise and research. Taylor admits these difficulties while holding to the inherent, conventional wisdom of the concept (Taylor, 1987). He claims for it the value of a risk factor both for susceptibility to disease and for response to psychotherapy. The presence of alexithymia in doctors would presumably make collusion with somatisation easier.

\section{Conditions embraced by somatisation}

Despite the history of frequent changes in terminology and the fact that we have ended up with three overlapping categorisations of somatisation; ICD10, DSM-IV, and the folkloric language which doctors use in their communication with each other, the clinician is likely to have in mind three enduring terms and concepts when faced with somatisation. These are hysteria, hypochondriasis and neurasthenia, and it is important that their history be understood. 
The phenomena embraced by the term 'hysteria' have been regarded as a distinct syndrome for millennia, and the syndrome, its name and its theoretical constructs have provided in the last 100 years the arena in which patients and doctors alike played out the debate about somatisation. Hysteria, like its parent term 'neurosis', has now been largely excluded from the international classifications, but not from doctors' and patients' everyday language. Such a disjunction has major implications.

Although there were implications of psychogenicity in Sydenham's 17th-century view that "the mind sickens more than the body", it was not until Freud proposed that conversion of psychic conflict into physical and mental symptoms by means of mental dissociation was at work in neurosis that psychogenetic thinking became fashionable. Early classifiers split hysteria into dissociative disorders (of memory, consciousness and personality) and conversion disorders (of somatic function), but this division is now blurred. For instance, ICD-10 gives priority to dissociation over conversion, and uses that term to replace hysteria.

The term 'hypochondriasis' fell out of use in the last half of the 19th century, its components being dispersed to other psychiatric diagnoses. Now it has returned, though not yet clearly distinguished from depression and anxiety, nor indeed from the other somatoform disorders. It is defined as belief or attitude; ICD-10 describes it as a persistent preoccupation with the possibility of having one or more serious and progressive physical disorders. It thus represents a particular cognitive set which has certain behavioural consequences, but it often involves a somatic experience of some sort. There is often an element of hypochondriasis in other somatoform disorders. A useful distinction between acute and chronic types can be made (Robbins \& Kirmayer, 1996).

'Neurasthenia' was an important 19th-century term which the American, Beard, used to draw together different types of neurosis, particularly those in which the patient had an aggregate of physical and psychological symptoms. It is a term still used in many non-Western countries and appears in ICD-10 where it is defined as either a complaint of increased fatigue after mental effort, or of feelings of bodily or physical weakness and exhaustion after only minimal effort. Recent studies suggest that it is not a distinct entity, but rather a continuum, and that it has a high degree of association with other psychiatric illnesses, especially anxiety and depression. It describes chronic fatigue syndrome which, although difficult to define, has become the test case to prove or disprove psychogenicity of a physical disorder, replacing repetitive strain injury as the arena for public positioning of polarised views about medically unexplained symptoms.

\section{Assessment}

\section{Biopsychosocial assessment}

The somatiser is fixated on the biological. To widen the agenda takes time, patience and educational skills. Referral to the psychiatrist will usually have been made with difficulty and against resistance, perhaps sold as being to a psychiatrist who is 'different'. Refer to Box 1 for some relevant questions. The patient may be unclear as to whether or not the psychiatrist is a doctor, and if so, what expectations they can expect to have about familiarity with the physical aspects of their presentation. Models encompassed by the psychiatrist may be questioned, especially with respect to alternative medicine. These are tricky matters to handle. Somatisers often demand practical answers, but the psychiatrist needs to be alert to what others have missed, and to do this needs to listen to the patient's narrative. Premature closure of this may destroy the chance to detect that one slip of the tongue, that particular construct, or those many other sorts of subtle clues which allow the psychiatrist in to areas from which others have been excluded. A useful strategy is to ask the patient, "When were you last really well?" They will often say, "Do you mean mentally or physically?" This provides the chance to remind the patient that you are interested in their construct, and in exploring their attitudes. They have raised the issue of the mind/body split, and reminding them of this gives them a chance to educate the psychiatrist about their own mind-set and culture; their belief system

Box 1. Questions for use in assessment of somatisers

What is your understanding of why you were referred?

How did you feel about that?

What do you expect from this referral?

When were you last really well?

Of all the things that worry you, what worries you most?

What is your understanding of how you came to be the way you are? 
and practices, and the way in which illness is constructed within that environment (Box 2).

In any event, such matters should be dealt with in a way that will encourage the patient to see that you have respect for their construct of the illness, that you acknowledge that you are dealing with unexplained symptoms, and that you are interested in working toward a joint understanding of the meaning and significance of the sickness rather than dismissing them as having nothing wrong, as others have done. The attitude displayed during these negotiations will reveal the cognitive set of the patient and help determine which type of somatisation predominates, and will guide the rest of the assessment. Is there any sign at all of psychological mindedness that will permit a joint exploration of the possibility of mind-caused factors being relevant?

It is more likely than not that the psychiatrist will perform a physical examination, at least of the relevant parts, if this seems natural in the situation; such examination often throws light on the cognitive set and will act as a reminder to consider drug and alcohol abuse.

An interview with family members will usually be required; they may or may not be somatisers, and they may be compulsive carers. In complex cases data will have to be gathered from a number of sources. It is useful to test hypotheses with these other sources, sharing with them the uncertainty. A multidisciplinary assessment involving all relevant doctors is common, and indeed often it is this process that brings to light the gap which clinches the psychodynamic formulation. For example:

Box 2. Some golden rules for assessing somatisation

Know where you stand on the theoretical issues involved

Find common ground for completing the assessment

Instil hope but not the promise of cure

Ferret out all possible treatable primary and secondary psychiatric and physical conditions

Listen with the psychiatrist's ear to the patient's narrative

Negotiate a formulation with all concerned, including the patient

Educate all concerned, including the patient, about the mechanisms involved
Mrs $\mathrm{H}$ is a 45-year-old woman with ulcerative colitis. She infuriates the medical and nursing staff with her excessive vigilance over her treatment. The psychiatrist has acted as broker for years. Only when she developed breast cancer and accepted treatment of that with equanimity was it possible to understand that she had a differential perception of her illnesses based on the concept described by Freud in his writing on the death instinct, wherein he argued that each person has an unconsciously determined preferred way of dying, and opposes every other way. It now became possible to work with the patient on the meaning of her ulcerative colitis, and achieve a more harmonious relationship with staff.

At some point the patient will expect education about how the psychiatrist views the problem. Somatisers do not handle uncertainty well, and the formulation and its delivery must allow for this.

\section{Formulation}

Formulation, or the answer to the question, "Why is this patient ill in this way at this time?", requires negotiation with the patient, the family, and the other clinicians involved. The biopsychosocial model is the basis, but it needs spelling out in a multicausal, interactive aetiological model such as that espoused by Mayou et al (1995b) and illustrated in Table 1.

Key aetiological mechanisms should be spelt out in detail, and an explanation of how this will provide a rational basis for management should be given. This is a critical point, and if not negotiated well, the patient is likely to withdraw. In particular, the distinction between dissociative (conversion) and psychophysiological mechanisms needs to be made. Demonstration of the effects of hyperventilation may be a useful strategy. Likening the tendency to somatise to the holding of other personality traits and cultural beliefs is a way of helping the patient conceptualise the process. Of course, the language must be appropriate to the

Table 1. A grid for recording and classifying the multicausal factors of the interactive aetiological model, with examples (after Sharpe et al, 1995)

\begin{tabular}{|c|c|c|c|}
\hline & Biological & Psychological & Social \\
\hline Predisposing & Genetic & $\begin{array}{l}\text { Somatising } \\
\text { personality }\end{array}$ & $\begin{array}{l}\text { Cultural } \\
\text { attitudes }\end{array}$ \\
\hline Precipitating & $\begin{array}{c}\text { Physical } \\
\text { illness }\end{array}$ & Stress & Job loss \\
\hline Perpetuating & $\begin{array}{l}\text { Psychotropic } \\
\text { drug side- } \\
\text { effects }\end{array}$ & $\begin{array}{l}\text { Depressive } \\
\text { illness }\end{array}$ & $\begin{array}{l}\text { Sick role } \\
\text { rewards }\end{array}$ \\
\hline
\end{tabular}


situation, and the lesson is unlikely to be learned in one sitting.

\section{Management}

A positive attitude to management is necessary and is justifiable. Improvement in functional outcome and decrease in health care utilisation and costs can be expected with even the simplest of interventions (Box 3 ).

\section{Acknowledgement}

Acknowledging to oneself that somatisation is a cultural norm likely to be directing the doctor's thinking as much as the patient's is the first step required. Some doctors are more prone to that way of thinking than others. Goldberg and colleagues (1989) have shown that it is possible to re-train doctors to treat somatisation, but not all doctors are so educable (Cohen-Cole et al, 1991). Acknowledgement to the patient that both parties are confronting a highly valued way of thinking with roots largely inaccessible to patient and doctor alike is also required.

Box 3. Some golden rules for managing somatisation

Negotiate a management plan with all involved, including the patient

Agree about who will be the case manager, and offer support to that person

Give the patient a role in case management by use of diaries and patient-held case records

Base treatment on evidence-based recommendations: treatment of comorbid physical and psychiatric conditions; use of Smith et al's (1995) management plan for primary physicians; cognitivebehavioural psychotherapy individually or in groups; modified psychodynamic and interpersonal therapy for selected conditions; involve families and ethnic health workers

Use a multidisciplinary approach, and recognise that an allied health professional may be the most acceptable key therapist

\section{Case management}

Once the formulation is agreed to by all parties including the patient, interventions and their coordinated management should be negotiated. Someone must be the case manager (Barsky, 1996). It is difficult for the psychiatrist to play this role; somatisation is a powerful process which can push the psychiatrist's comfort with physical medicine to the limits. The case manager needs to be someone who feels comfortable with uncertainty and is prepared to take the risk of non-investigation. The psychiatrist has a major role to play in supporting such a case manager and counselling the patient about how to handle their various doctors. A single letter from a psychiatrist to a primary care physician, explaining the diagnosis and suggesting a rational management plan, can have an extraordinary impact (Smith et al, 1995). The use of a patientheld case record can help minimise confusion and conflict.

\section{Specific interventions}

If somatisation is an episodic component of a reactive disorder, resolution can be expected. Treatment of underlying depression or anxiety will be required, with explanation to all concerned that the somatisation is largely reactive but requires firm but supportive handling. A major role for the psychiatrist in such cases is to help prevent the condition becoming chronic, and to help prevent iatrogenic physical complications.

If somatising traits dominate the picture, it will be important to dissect out primary and secondary effects (Robbins \& Kirmayer, 1996). Medication will be needed for severe anxiety and depression, and is of proven value in chronic pain. Although antidepressants are often used for the treatment of the depressive component of neurasthenia, their efficacy is not proven (Vercoulen et al, 1996). Since chronic somatising patients are more likely than not to have comorbid physical disorder, iatrogenic or otherwise, and certainly likely to be on multiple and diverse drugs including analgesics, the psychiatrist is often operating beyond the boundaries of evidence-based medicine in prescribing psychotropic drugs in such situations. Addition of psychotropic medication to that pot is a potentially hazardous exercise requiring close monitoring and coordination (Jachna et al, 1996). Involving the pharmacist helps. The tendency of the patient to experience side-effects at low 
threshold levels and to incorporate them into their somatising mind set is a further challenge.

\section{Physical treatments}

Patients may already be undertaking physiotherapy, relaxation therapy, or similar but more marginal therapies. These may be specifically indicated, as in the presence of anxiety symptoms, or their use may be a non-specific correlate of the fact that the patient is a somatiser and finds physical attention appropriate. Used wisely, the physical therapists can become major allies in the management plan, acceptable therapists who are able to contain the situation.

\section{Psychotherapeutic interventions}

Psychotherapeutic intervention for a psychological problem seems to have such face validity that one might expect there to be considerable evidence-based guidelines available. In the case of those patients likely to be seen by psychiatrists, this has not been so until recently. Somatisers tend to be so chronic and complex that it is difficult to design appropriate studies and recruit satisfactorily. Nevertheless, we have clues about what therapies are worth trying in certain groups of patients. They include cognitive-behavioural and psychodynamic therapies.

Barsky (1996) has reviewed the cognitiveeducational techniques which seem useful in the management of chronic hypochondriasis. Group therapy involves didactic presentation, experiential learning and discussion. A similar programme for patients with somatisation disorder yielded significant improvement in physical and mental health and decrease in health care costs (Kashner et al, 1995).

There are recent evidence-based demonstrations of the efficacy of a cognitivebehavioural approach in a number of conditions, including pain, irritable bowel syndrome and dyspepsia, reviewed by Guthrie (1996), and for chronic fatigue syndrome (Sharpe et al, 1996). Psychodynamic therapy appears to be effective in the treatment of irritable bowel syndrome (Guthrie et al, 1991).

Replication has been a problem, and this raises the possibility that what really works is a confident team, dedicated to helping patients make some sort of sense of why something that seems so naturally physical to them responds best to psychosocial interventions that seem so unnatural to them. Many of these issues are discussed extensively in a recent book on the topic (Mayou et al, 1995a).

\section{References}

American Psychiatric Association (1994) Diagnostic and Statistical Manual of Mental Disorders (4th Edn) (DSM-IV). Washington: American Psychiatric Association.

*Barsky, A. J. (1996) Hypochondriasis. Medical management and psychiatric treatment. Psychosomatics, 37, 48-56.

Bass, C. (1994) Somatisation disorder: the need for effective intervention studies. General Hospital Psychiatry, 16, 379-380.

-_ \& Murphy, M. (1995) Somatoform and personality disorders: syndromal comorbidity and overlapping developmental pathways. Journal of Psychosomatic Research, 39, 403-427.

Berrios, G. \& Porter, R. (eds) (1995) A History of Clinical Psychiatry. London: Athlone.

Cohen-Cole, S. A., Howell, E. F., Barrett, J. E., et al (1991) Consultation-liaison research: four selected topics. In Handbook of Studies on General Hospital Psychiatry (eds F. K. Judd, G. B. Burrows \& D. R. Lipsitt), pp. 79-98. Amsterdam: Elsevier.

Gill, D. \& Bass, C. (1997) Somatoform and dissociative disorders: assessment and treatment. Advances in Psychiatric Treatment, in press.

*Goldberg, D., Gask, L. \& O'Dowd, T. (1989) The treatment of somatisation: teaching techniques of reattribution. Journal of Psychosomatic Research, 33, 689-695.

Guthrie, E. (1996) Emotional disorder in chronic illness: psychotherapeutic interventions. British Journal of Psychiatry, 168, 265-273.

-, Creed, F. H., Dawson, D., et al (1991) A controlled study of psychological treatment of the irritable bowel syndrome. Gastroenterology, 100, 450-457.

Hiller, W., Rief, W. \& Fichter, M. M. (1995) Further evidence for a broader concept of somatisation disorder using the somatic symptom index. Psychosomatics, 36, 285-294.

Jachna, J. S., Lane R. D. \& Gelenberg, A. J. (1996) Psychopharmacology. In Textbook of Consultation-Liaison Psychiatry (eds J. R. Rundell \& M. G. Wise), pp. 958-1005. Washington: American Psychiatric Press.

*Kashner, T. M., Rost, K., Cohern, B., et al (1995) Enhancing the health of somatisation disorder patients. Effectiveness of short-term group therapy. Psychosomatics, 36, 462-470.

Kirmayer, L. J., Robbins, J. M. \& Paris, J. (1994) Somatoform disorders: personality and the social matrix. Journal of Abnormal Psychology, 103, 125-136.

Lipowski, Z. J. (1968) Review of consultation psychiatry and psychosomatic medicine: III. Theoretical issues. Psychosomatic Medicine, 30, 394-422.

- (1988) Somatisation: the concept and its clinical application. American Journal of Psychiatry, 145, 1358-1368.

Mayou, R. (1996) Accident neurosis revisited. British Journal of Psychiatry, 168, 399-403.

*-, Bass, C. \& Sharpe, M. (eds) (1995a) Treatment of Functional Somatic Symptoms. Oxford: Oxford University Press.

- - _ \& - (1995b) Overview of epidemiology, classification, and aetiology. In Treatment of Functional Somatic Symptoms (eds R. Mayou, C. Bass \& M. Sharpe), pp. 42-65. Oxford: Oxford University Press.

Menninger, W. C. (1947) Psychosomatic medicine: somatization reactions. Psychosomatic Medicine, 9, 92-97.

Pilowsky, I. (1978) A general classification of abnormal illness behaviours. British Journal of Medical Psychology, 51, 131-137.

Robbins, J. M. \& Kirmayer, L. J. (1996) Transient and persistent hypochondriacal worry in primary care. Psychological Medicine, 26, 575-589.

Rogers, M. P., Weishenker, N. J., Warshaw, M.G., et al (1996) Prevalence of somatoform disorders in a large sample of patients with anxiety disorders. Psychosomatics, 37, 17-22. 
Sharpe, M., Bass, C. \& Mayou, R. (1995) An overview of the treatment of somatic symptoms. In Treatment of Functional Somatic Symptoms (eds R. Mayou, C. Bass \& M. Sharpe), pp. 66-86. Oxford: Oxford University Press.

*-, Hawton, K., Simkin, S. S., et al (1996) Cognitive-behaviour therapy for the chronic fatigue syndrome: a randomised controlled trial. British Medical Journal, 312, 22-26.

Singh, B., Nunn, K., Martin, J., et al (1981) Abnormal treatment behaviour. British Journal of Medical Psychology, 54, 131-137.

*Smith, G. R., Rost, K. \& Kashner, T. M. (1995) A trial of the effect of a standardised psychiatric consultation on health outcomes and costs in somatising patients. Archives of General Psychiatry, 52, 238-243.

Taylor, G. J. (1987) Psychosomatic medicine and contemporary psychoanalysis. Stress and Health Series Monograph 3 (ed. G. Goldberger). Madison: International Universities Press.

Vercoulen, J. H., Swanik, C. M., Zitman, F. G., et al (1996) Randomised, double-blind, placebo-controlled study of fluoxetine in chronic fatigue syndrome. Lancet, 347, 858-861.

World Health Organization (1992) The Tenth Revision of the International Classification of Mental and Behavioural Disorders (ICD-10). Geneva: World Health Organization.

Wotton, H. (1642) Upon the death of Sir Albert Morton's wife. In Familiar Quotations by John Bartlett, 14th edn (1968) (ed. E M. Beck), p. 300. London: MacMillan.

* indicates articles of particular interest

\section{Multiple choice questions}

\section{Somatisation:}

a is equivalent to ICD-10 somatoform disorder

b is equivalent to DSM-IV somatoform disorder

c is an ubiquitous tendency present in all cultures

d involves experiential, cognitive and behavioural elements

e is most likely to be found in comorbidity with other psychiatric and physical disorders.

2. Concepts related to somatisation include:
a medically unexplained symptoms
b abnormal illness behaviour
c functional symptoms
d alexithymia
e organic mental disorder.

3. Conditions embraced by somatisation include:
a neurasthenia
b delirium
c dissociative disorder
d psychophysiological disorder
e hypochondriacal disorder.

4. Assessment of somatisation is likely to involve:
a negotiation to find common ground
b physical examination by the psychiatrist
c discouraging of involvement of family members
d negotiation of a formulation
e certainty of diagnosis.

5. In the management of somatisation:
a no evidence-based therapies are available
b medication is unlikely to be helpful
c management should be negotiated with all concerned
d the use of a case manager other than a psychiatrist is desirable
e the role of the psychiatrist should be confined to that of diagnostician.

\begin{tabular}{|c|c|c|c|c|}
\hline \multicolumn{5}{|c|}{ MCQ answers } \\
\hline 1 & 2 & 3 & 4 & 5 \\
\hline a $F$ & a $T$ & a $\mathrm{T}$ & a $\mathrm{T}$ & a $F$ \\
\hline b F & b $T$ & b F & b $\mathrm{T}$ & b $F$ \\
\hline c $\mathrm{T}$ & c $\mathrm{T}$ & c $\mathrm{T}$ & c F & c $\mathrm{T}$ \\
\hline d $\mathrm{T}$ & d $T$ & d $T$ & d $T$ & d $\mathrm{T}$ \\
\hline e $F$ & e $F$ & e $\mathrm{T}$ & e F & e $F$ \\
\hline
\end{tabular}

